\title{
SENSIBILIDADE DO MODELO DE FRAGILIDADES AMBIENTAIS À PONDERAÇÃO MULTICRITERIAL: ASPECTOS FÍSICOS DA BACIA HIDROGRÁFICA DO ALTO URUGUAI
}

\author{
Damáris Gonçalves Padilha ${ }^{1}$, Mário Luiz Trevisan ${ }^{2}$, Jussara Cabral Cruz ${ }^{3}$ \\ ${ }^{1}$ Eng $^{\mathrm{a}}$. Florestal, M.Sc., UFSM, Santa Maria, RS, Brasil - damarispadilha@ gmail.com \\ ${ }^{2}$ Eng. Eletricista, Dr., UFSM, Santa Maria, RS, Brasil - eletroduto@gmail.com \\ ${ }^{3} \mathrm{Eng}^{\mathrm{a}}$. Civil, Dr ${ }^{\mathrm{a}}$., UFSM, Santa Maria, RS, Brasil - jussaracruz@gmail.com \\ Recebido para publicação: 30/04/2013 - Aceito para publicação: 12/03/2014
}

\begin{abstract}
Resumo
Modelos de fragilidades ambientais compreendem, de maneira geral, a avaliação de $n$ aspectos (ou fatores) relativos às características locais, usualmente tratadas por técnicos de diferentes áreas constituintes de uma equipe multidisciplinar. Porém a definição dos $n$ aspectos por parte da equipe nem sempre acontece de forma unânime, não raro ocorrendo, no início do processo de avaliação, dúvidas sobre o quanto realmente cada aspecto ou fator (indicador) apresenta-se significativo dentro do modelo de avaliação. Como estudo de caso, foi realizada a sensibilidade das ponderações dos fatores que compõem o método multicriterial AHP (Analytic Hierarchy Process), aplicado na região da bacia hidrográfica do Alto Uruguai. A metodologia consistiu na ponderação sistemática de cada fator constituinte do modelo para posterior consideração quanto a sua influência em relação ao conjunto dos fatores, proporcionando uma avaliação quanto a sua eficácia na identificação de pesos dos fatores em cenários que mostram maior discriminância no conjunto, além de apresentar alternativas na composição dos pesos. Assim, a partir dos resultados da análise, configura-se a importância da realização do estudo da sensibilidade em modelos de fragilidade ambiental aplicados à bacia hidrográfica.

Palavras-chave: Análise de sensibilidade; avaliação ambiental; multicritério.
\end{abstract}

\begin{abstract}
Sensitiveness of model to environmental fragilities to multicriterial weighting: physical aspects of watershed of the Alto Uruguay. Environmental fragilities models comprise, generally, the evaluation of $n$ criteria (or factors) on local characteristics, usually handled by technicians from different areas in a multidisciplinary team. However, definition of $n$ aspects by team does not always happen unanimously, often occurring early in the evaluation process doubts whether every aspect or indicator is in fact significant within the evaluation model. As a case study, it was performed the sensitivity of weighting factors that comprising the multicriterial method AHP (Analytic Hierarchy Process) applied to the basin of the Alto Uruguay region. After evaluating the multicriterial method, we applied the sensitiveness analysis to improve the weighting process. Therefore, from the results of this analysis, it sets up the importance of the study of sensitiveness of environmental fragility models for the watershed better understanding.

Keywords: Sensitiveness analysis; environmental assessment; multicriterial weighting.
\end{abstract}

\section{INTRODUÇÃO}

Os modelos de análise de fragilidades ambientais, buscando determinar a capacidade dos sistemas naturais de suportarem as intervenções humanas, compreendem, de maneira geral, a avaliação de $n$ fatores relativos às características locais, usualmente tratadas por técnicos de diferentes áreas constituintes de uma equipe multidisciplinar. Cabe à equipe ou ao projetista, identificar, no momento do diagnóstico, quais serão os $n$ fatores que farão parte da avaliação em cada um dos ambientes, assim como a definição de sua importância relativa dentro do modelo. Naturalmente, isso remete a problemas de unanimidade na seleção dos fatores. Santos (2004) afirma que, para todo problema apontado no decorrer de um planejamento, há sempre uma ou mais alternativas de solução, cabendo ao planejador encontrar 
estratégias para prover medidas da distância entre o que é perfeitamente certo e o que é completamente errado, apresentando assim as múltiplas alternativas para a tomada de decisão.

Para Santos (2004), entre diversos métodos dirigidos à tomada de decisão, a análise multicriterial apresenta-se bastante adequada para aplicação em planejamentos ambientais. Uma das características da Avaliação Multicritério (AMC) para modelos ambientais é a de contemplar a diversidade natural em $n$ fatores e considerar a sua variabilidade, atribuindo a eles pesos de importância dentro de cada composição do modelo. No entanto, é a análise de sensibilidade da ponderação atribuída aos $n$ fatores que permite o seu aperfeiçoamento, com a inclusão de técnicas estatísticas para a avaliação da recomposição das importâncias entre os aspectos na construção do modelo conceitual.

Em estudos específicos realizados em escala de bacias hidrográficas, a utilização dessas metodologias tem buscado minimizar possíveis erros que ocorrem no momento de determinar a importância de cada aspecto que irá compor a avaliação dos impactos ambientais decorrentes da implantação de um empreendimento.

O inventário de recursos hídricos realizado pela Universidade Federal de Santa Maria (UFSM) (2002) utilizou a análise multicritério ambiental com o objetivo de eliminar regiões ambientalmente inadequadas para a implantação das obras de barramento para o fomento de atividades de irrigação no estado do Rio Grande do Sul. A aplicação de um método multicritério em problemas de tomada de decisão na gestão de recursos hídricos é uma tarefa bastante delicada, segundo Zuffo (1998), pois depende da familiaridade do decisor com a metodologia adotada e da natureza das informações disponíveis.

Nessa linha de pesquisa, a Universidade Federal de Santa Maria (UFSM) e a Universidade do Pampa (UNIPAMPA) (2011) aplicaram método multicritério AHP (Analytic Hierarchy Process), com o objetivo de aprimorar metodologias de avaliação de fragilidades ambientais em apoio à gestão ambiental integrada e à tomada de decisão no processo de licenciamento ambiental de empreendimentos hidrelétricos nas regiões do Alto e Médio Rio Uruguai em território brasileiro.

Verificada a importância dessa metodologia de avaliação, como estudo de caso, objetiva-se realizar a sensibilidade das ponderações dos fatores que compõem o método aplicado pelos autores, proporcionando uma avaliação quanto à sua eficácia na identificação dos fatores mais ou menos sensíveis, além de apresentar alternativas para a composição dos pesos.

\section{Seleção dos indicadores do modelo}

O interesse pelo uso de indicadores ambientais como subsídio à tomada de decisão tem crescido mundialmente (FIDALGO, 2003), porque consistem de grandezas mensuráveis que permitem declarações de uma estrutura complexa (LANG; BLASCHKE, 2009). O uso do termo "indicadores" tornou-se mais frequente nos últimos anos, com a popularização do conceito de sustentabilidade e a necessidade de ferramentas para sua mensuração e monitoramento (FERRAZ et al., 2009).

A Organisation for Economic Co-operation and Development (OECD, 2011) define indicador como "parâmetro, ou valor calculado a partir de parâmetros, fornecendo indicações sobre ou descrevendo o estado de um fenômeno, do meio ambiente ou de uma zona geográfica", e lhes atribui a função de reduzir o número de medidas e de parâmetros que seriam normalmente necessários para analisar uma situação com "exatidão". Há, portanto, a premissa de que esse parâmetro esteja associado a um atributo ou a um conjunto de atributos essenciais para tomada de decisão, os quais possam sintetizar as características do sistema sob análise e não apenas caracterizem feições de forma isolada. Para Santos (2007), os indicadores decodificam estados do meio, como expressões de um estado natural, de uma autorregulação ou de reação a uma ação qualquer, e apresentam grande importância na tomada de decisão.

Na seleção dos indicadores de um modelo conceitual, seja para definição de uma fragilidade natural ou do resultado da ação de um empreendimento, é importante o conhecimento prévio da área a ser analisada, dos objetivos do modelo e disponibilidade dos dados que abranjam toda a área. Deve-se ainda observar a importância de um indicador no contexto integrado do modelo, uma vez que ela varia conforme o objetivo da avaliação ambiental. Esse processo de seleção dos dados a serem trabalhados em um modelo pode ser considerado a fase mais importante no momento da sua elaboração, uma vez que, ao representar sua estrutura, as informações decorrentes do processo seletivo dependerão da representatividade singular de cada indicador e da sua inter-relação com os demais indicadores. 
A seleção de variáveis indicadoras é de certa forma uma arte, pois envolve, além de boa ciência e boa técnica, criatividade para buscar no universo de dados disponíveis aqueles que atendem simultaneamente aos requisitos de resposta ao estado de padrões e processos significativos, apresentam-se em escala compatível com a modelagem e o tamanho da área em estudo e são disponíveis para $100 \%$ da área (UFSM; UNIPAMPA, 2011). Entretanto, apesar de reconhecer que o sucesso de um planejamento depende dos temas escolhidos, Santos (2004) destaca que é muito raro encontrar justificativas para seleção de indicadores e o conteúdo de cada um deles. A disponibilidade dos dados tem sido, na prática, o fator decisório no momento da escolha dos temas que farão parte da análise e formulação de um planejamento.

Em um ambiente natural ocorrem inter-relações e interdependências entre os diferentes aspectos existentes, entretanto, para efeito de estudos, há a necessidade de separar, agrupar e/ou reagrupar determinadas características conforme a especificidade das áreas de conhecimento.

Santos (2004) salienta que é comum agrupar indicadores que se destinam a expressar as condições de qualidade ou estado do meio, sendo que, algumas vezes, os indicadores são organizados pela natureza da informação, como, por exemplo, fisiográfica, topográfica, cultural, econômica e/ou legal. Para Fidalgo (2003), os indicadores são agrupados para formar um sistema que serve como base para a avaliação do estado vigente e do desenvolvimento do sistema analisado.

Uma maneira de prevenir os impactos sobre as áreas de preservação ambiental diz respeito, primeiramente, ao entendimento sobre as causas, as intensidades e principalmente a consequência desses impactos causados no ambiente natural. Maganhotto et al. (2011) salientam que, para isso, é necessário o conhecimento das suas variáveis (indicadores) e suas especificidades. Para Ross (1994). as unidades de fragilidade dos ambientes naturais devem ser resultantes dos levantamentos básicos de geomorfologia, solos, cobertura vegetal/uso da terra e clima. Esses elementos, tratados de forma integrada, possibilitam obter um diagnóstico das diferentes categorias hierárquicas da fragilidade dos ambientes naturais.

\section{Descrição da área de estudo}

Integrante da bacia hidrográfica do rio Uruguai em território brasileiro, a bacia hidrográfica do Alto Uruguai (ou Uruguai Alto) localiza-se nos estados do Rio Grande do Sul e Santa Catarina, entre as coordenadas $49^{\circ} 17^{\prime} 13^{\prime \prime} \mathrm{W}$ e $53^{\circ} 52^{\prime} 31^{\prime \prime} \mathrm{W}$ e $28^{\circ} 46^{\prime} 36^{\prime \prime} \mathrm{S}$ e $26^{\circ} 14^{\prime} 02^{\prime \prime} \mathrm{S}$. Essa bacia compreende os dois principais tributários formadores do rio Uruguai em território brasileiro, o rio Canoas e o rio Pelotas, e apresenta uma área de $76.209 \mathrm{~km}^{2}$, relevo declivoso e cotas altimétricas variando de 160 a $1800 \mathrm{~m}$. De acordo com MMA (2006), a qualidade da água na bacia hidrográfica do rio Uruguai pode ser considerada boa, principalmente quando comparada à realidade de outras regiões hidrográficas do país.

\section{MATERIAL E MÉTODOS}

A análise de sensibilidade descrita neste trabalho é parte integrante do processo composto por duas etapas técnicas e uma etapa decisória. A primeira consiste na escolha das variáveis ou fatores que irão contemplar o modelo de análise e a definição da fragilidade de cada fator frente ao empreendimento a ser inserido, bem como a atribuição da ponderação dos fatores e aspectos pela avaliação multicritério. Essa primeira etapa foi realizada por UFSM; UNIPAMPA (2001).

Esse processo resulta em um cenário de fragilidade referente ao aspecto ou ao conjunto dos aspectos analisados, considerada a ponderação definida pela equipe multidisciplinar. O cenário obtido pela ponderação da equipe multidisciplinar pode resultar em duas situações: uma que atende os requisitos técnicos, ou seja, o cenário resultante representa plenamente o propósito para o qual foi elaborado, passando para a etapa decisória. Em não atendendo, aplica-se o modelo de sensibilidade objeto deste estudo (etapa destacada na Figura 1), que consiste em correlacionar cenários ponderados (sistematicamente) com o cenário homogêneo (ponderação igualitária para todos os fatores), descrito adiante no item "Cenário homogêneo ou cenário testemunha", elencando cenários que atendam os requisitos, uma vez que as ponderações advindas da análise de sensibilidade aumentam a discriminância de seus fatores, passando então à etapa decisória.

A abordagem metodológica da análise da sensibilidade foi aplicada para os aspectos físicos, bióticos e antrópicos integrantes do modelo de fragilidade realizado por UFSM; UNIPAMPA (2001). Em 
função do grande volume de informações (dados), optou-se por apresentar apenas os resultados obtidos da análise dos aspectos físicos, deixando para uma etapa posterior a apresentação dos demais resultados.

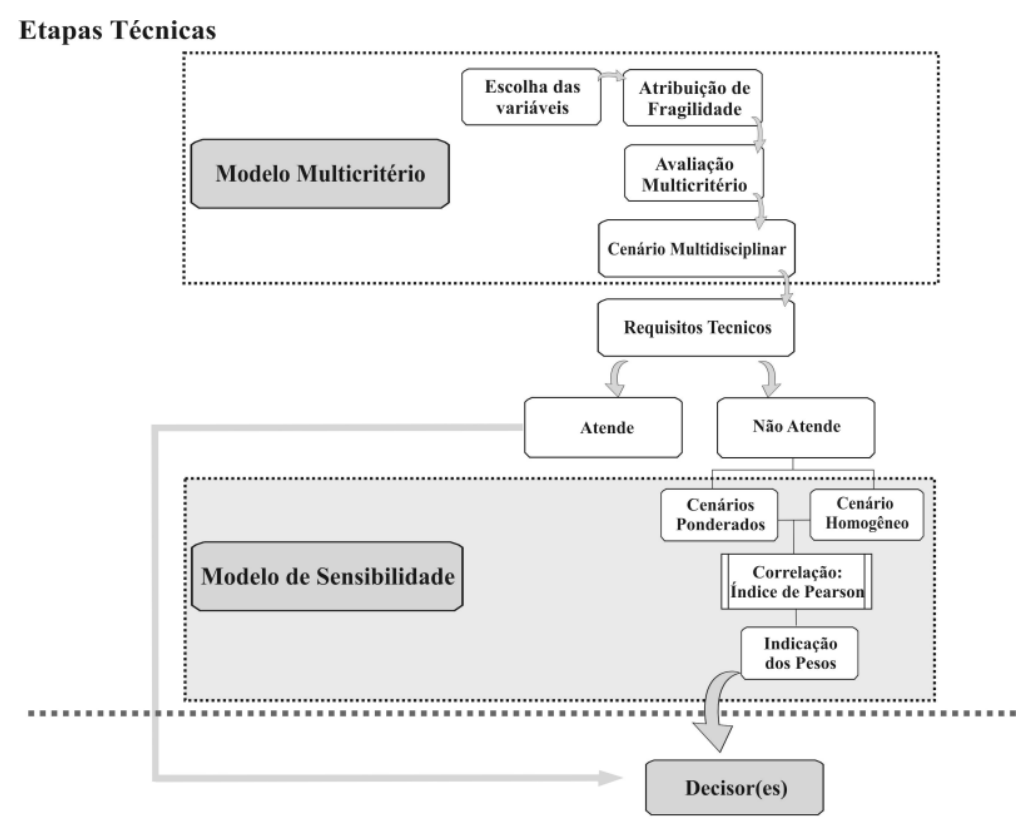

Etapa Decisória

Figura 1. Fluxograma para aplicação do modelo de sensibilidade.

Figure 1. Flowchart for sensitiveness model applying.

\section{Aspectos físicos da bacia hidrográfica}

Os aspectos e fatores físicos utilizados nesta avaliação foram definidos por UFSM e UNIPAMPA (2011), levando em consideração a fragilidade ambiental para implantação de empreendimentos hidrelétricos na área de estudo. Os autores desenvolveram, para a região da bacia, uma metodologia de avaliação ambiental integrada aplicada ao processo de análise de viabilidade desses empreendimentos sobre o seu território. Com essa finalidade, atribuíram valores de fragilidade ambiental para cada fator. Em ambiente de geoprocessamento, os mapas digitais (rasters) correspondentes aos fatores tiveram seus valores de fragilidade uniformizados na escala de 0 a 255 , a fim de torná-los comparáveis. Os fatores foram agrupados pelos autores em 3 níveis de avaliação - variáveis (ou fatores), aspectos e blocos temáticos - em ordem crescente de abrangência na modelagem, sendo os aspectos definidos como o resultado da combinação linear ponderada dos fatores que apresentam padrões ou processos fortemente relacionados. A mesma lógica segue-se para a formação dos blocos temáticos com a soma ponderada dos respectivos aspectos.

Para formação do bloco físico, foram considerados por UFSM/UNIPAMPA (2011) os seguintes aspectos: geologia e geomorfologia, estabilidade de encostas, contaminação das águas, fragilidade à erosão e áreas de mineração. A tabela 1 apresenta a importância atribuída para os aspectos físicos da bacia hidrográfica e os fatores que foram associados para sua construção. Uma descrição minuciosa da composição dos fatores e da construção dos aspectos e bloco podem ser encontrados em UFSM/UNIPAMPA (2011).

\section{Avaliação multicritério (AMC)}

Em um processo de avaliação ambiental integrada, uma das fases de fundamental importância é a construção do modelo de avaliação multicritério, pois normalmente os participantes desse processo revelam posições relativas quanto à interpretação da importância de cada aspecto integrante. Diante do conhecimento parcial da realidade e da sua subdivisão, do conhecimento leigo e sua interpretação, da predominância das incertezas na fase inicial de construção do modelo, as importâncias dadas para os $n$ fatores ainda não estão em condições de serem tecnicamente definidas. 
Tabela 1. Aspectos físicos e fatores associados.

Table 1. Physical aspects and associated factors.

\begin{tabular}{|c|c|c|}
\hline Aspectos & Importância & Fatores associados \\
\hline $\begin{array}{l}\text { Geologia e } \\
\text { geomorfologia }\end{array}$ & $\begin{array}{l}\text { Determinação da qualidade da rocha, resistência ao } \\
\text { intemperismo, resistência mecânica, associadas à } \\
\text { declividade, originando diferentes modelados de } \\
\text { relevo. Avaliação de lineamentos, superfície } \\
\text { potenciométrica e zona vadosa associados à } \\
\text { probabilidade de impactos em aquíferos. }\end{array}$ & $\begin{array}{l}\text { - Geologia - litologia; } \\
\text { - Resistência dos solos; } \\
\text { - Água subterrânea; } \\
\text { • Aquífero; } \\
\text { - Superfície potenciométrica; } \\
\text { - Zona vadosa; } \\
\text { - Geomorfologia. }\end{array}$ \\
\hline $\begin{array}{l}\text { Estabilidade de } \\
\text { encostas }\end{array}$ & $\begin{array}{c}\text { Probabilidade de ocorrerem movimentos de massa e } \\
\text { áreas de risco. }\end{array}$ & $\begin{array}{l}\text { - Geologia - litologia; } \\
\text { - Declividade; } \\
\text { - Geomorfologia. }\end{array}$ \\
\hline $\begin{array}{l}\text { Contaminação } \\
\text { das águas }\end{array}$ & Qualidade das águas superficiais. & $\begin{array}{l}\text { - Carga orgânica remanescente das } \\
\text { populações; } \\
\text { - Carga orgânica das criações; } \\
\text { • Índice agrícola. }\end{array}$ \\
\hline $\begin{array}{l}\text { Fragilidade à } \\
\text { erosão }\end{array}$ & $\begin{array}{c}\text { Transporte de sedimentos aos leitos dos rios e } \\
\text { reservatórios. }\end{array}$ & $\begin{array}{c}\text { - Resistência dos solos; } \\
\text { - Classes de declividade; } \\
\text { - Incremento da fragilidade ao redor } \\
\text { dos cursos d'água de ordem maior } \\
\text { ou igual a } 3 \text {. }\end{array}$ \\
\hline $\begin{array}{l}\text { Áreas de } \\
\text { mineração }\end{array}$ & Contaminação das águas superficiais. & $\begin{array}{l}\text { • Títulos minerários; } \\
\text { - Incremento da fragilidade ao redor } \\
\text { das áreas de mineração; } \\
\text { - Incremento da fragilidade ao redor } \\
\text { dos cursos d'água de ordem maior } \\
\text { ou igual a } 3 \text {. }\end{array}$ \\
\hline
\end{tabular}

Fonte: Adaptado de UFSM/UNIPAMPA (2011).

Para Trevisan (2008), a avaliação multicritério é um processo construtivo no qual os resultados são utilizados como base para seu refinamento e, como tal, deve ser constantemente revisto, sendo que a mudança das características espaciais ou espacializáveis de uma bacia hidrográfica, no decorrer do tempo, é suficiente para justificar essa prática. Alguns trabalhos têm sido realizados com a aplicação da abordagem multicritério para avaliar e mapear fragilidades e riscos ambientais (VETTORAZZI et al., 2000; FERRAZ; VETTORAZZI, 2003; BOAS, 2005). No caso do método multicritério AHP, Boas (2006) o utilizou para o desenvolvimento de um modelo de auxílio aos tomadores de decisão nas questões relativas ao uso multipropósito da água, salientando que o método apresenta vários atributos desejáveis para esse tipo de estudo, a saber: é um processo de decisão estruturado que pode ser documentado e repetido; pode ser aplicado a situações que envolvem julgamentos subjetivos; utiliza tanto dados quantitativos como qualitativos; provê medidas de consistência das preferências, além de haver uma ampla documentação sobre suas aplicações práticas na literatura acadêmica.

Nesta abordagem, a palavra avaliação remete à atribuição de pesos - ou valoração - aos mapas temáticos representativos dos indicadores, denominados fatores, de modo que se construa um conjunto de mapas cujos pesos somam 1 (um), formando um vetor de valores, resultando num mapa-síntese (ou cenário) particular e único para aquele contexto, obtido pela aplicação da Equação 1.

$$
\mathbf{C}=\sum\left(\mathrm{F}_{\mathrm{i}} * \mathrm{w}_{\mathrm{i}}\right)=\mathrm{F}_{1} * \mathrm{w}_{1}+\mathrm{F}_{2} * \mathrm{w}_{2}+\ldots .+\mathrm{F}_{\mathrm{n}} * \mathrm{w}_{\mathrm{n}}
$$

Portanto, a obtenção de diferentes cenários (C) é resultante da variação do valor dos pesos (w) sobre os fatores $(\mathrm{F})$. 


\section{Análise de sensibilidade}

Para Trevisan (2008), é necessário avaliar a sensibilidade de métodos de avaliação multicritério como parte da ciência do apoio à decisão (consequentemente, da neutralidade científica) na construção de modelos, no processamento dos dados e na apresentação dos resultados.

$\mathrm{O}$ conceito de sensibilidade, para este estudo, considera as diferenças entre os fatores (mapas) resultantes de processamento da avaliação multicritério quando se faz a variação do peso de um fator mantendo-se os demais iguais. A figura 2 exemplifica de modo genérico esse processo. Nela são mostrados fatores $\left[F_{1}\right.$ a $\left.F_{n}\right]$, esquematizando-se uma variação de $F_{4}$ enquanto os demais são mantidos com pesos iguais entre si para cada etapa.

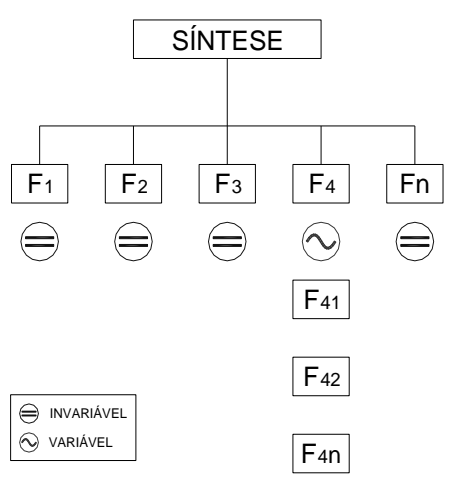

Fonte: Trevisan (2008).

Figura 2. Esquema da modelagem da sensibilidade.

Figure 2. Graph of modeling sensitiveness.

Os diferentes pesos em função do número de fatores são sintetizados e generalizados pela Equação 2. Essa equação determina o vetor de pesos, cujo somatório deve ser 1.

$$
w+\sum_{1}^{n f-1}[(1-w) /(n f-1)]=1
$$

em que: $\mathrm{w}=$ peso decimal atribuído ao fator $(\mathrm{w}=0,1$ a 0,5 , com intervalo de 0,1$)$;

$\mathrm{nf}=$ número total de fatores.

A análise de sensibilidade proposta por Trevisan (2008) fez evoluir significativamente o processo de seleção de fatores para análise multicritério (AMC), com sua inclusão revelando redundâncias entre fatores, evidenciando a importância dos fatores e reduzindo discussões supérfluas quanto à ponderação dos mesmos.

Para a realização dessa análise, se faz necessária a construção de um cenário comparativo, equivalente à testemunha nos estudos de experimentação das ciências agrárias, o qual foi denominado de cenário homogêneo $\left(\mathrm{C}_{\mathrm{H}}\right)$.

Cenário homogêneo ou cenário testemunha

Uma homogeneização, por conceito, consiste em atribuir importâncias iguais aos indicadores que compõem um modelo. Cenário homogêneo (ou cenário testemunha) é descrito estatisticamente como um cenário independente, o qual apresenta a distribuição equalitária da ponderação.

Embora essa situação não seja a desejada, por ser uma abstração matemática que não representa a diversidade, uma vez que coloca em igual relevância todos os aspectos da diversidade, o cenário homogêneo $\left(\mathrm{C}_{\mathrm{H}}\right)$ é fundamental pelo fato de que, na análise de sensibilidade, servirá de referencial para a avaliação da diversidade possível de ser revelada em outros cenários ponderados. Sua expressão matemática é dada pela unidade (1) dividida pelo número (n) de indicadores e parâmetros estabelecidos para o modelo específico (Equação 3).

$$
\mathrm{C}_{\mathrm{H}}=\mathrm{F}_{1} * 1 / \mathrm{n}+\mathrm{F}_{2} * 1 / \mathrm{n}+\ldots+\mathrm{F}_{\mathrm{n}} * 1 / \mathrm{n}
$$


em que: $\mathrm{C}_{\mathrm{H}=\text { cenário homogêneo; }}$

$\mathrm{F}_{1 \cdots \mathrm{n}}=$ indicador ou fator do modelo;

$\mathrm{n}=$ número de indicadores e fator do modelo.

Tendo-se elaborado o cenário testemunha, realiza-se um primeiro conjunto de comparações entre ele e os demais cenários sistematicamente ponderados, que serão abordados a seguir.

Cenários sistematicamente ponderados

Para cada conjunto de fatores, é realizada a distribuição dos valores das ponderações para o fator sob variação e para os demais, de modo a somarem sempre 1 (um), como pode ser observado na Equação 4.

$$
C_{n}=F_{p} * w_{v}+\sum_{i=1}^{n f-1}\left[F_{i} * w_{F}\right]
$$

em que: $\mathrm{C}_{\mathrm{n}}=$ cenários ponderados $(\mathrm{C} 1 \mathrm{a} \mathrm{C} 5)$;

$\mathrm{F}_{\mathrm{p}}=$ fator sob ponderação sistemática $\left[3 \leq \mathrm{F}_{\mathrm{p}} \leq 6\right]$;

$\mathrm{w}_{\mathrm{v}}=$ peso sistemático $(0,1$ a 0,5$)$;

$\mathrm{nf}=$ número total de fatores;

$\mathrm{F}_{\mathrm{i}}=$ cada um dos demais fatores;

$\mathrm{W}_{\mathrm{F}}=$ peso obtido pela equação 5 .

$$
w_{F}=\left[\left(1-w_{v}\right) /(n f-1)\right]
$$

A variação dos pesos é arbitrária, porém, para equilibrar tempo e volume de processamento versus preciosismo nos resultados, optou-se por variar os pesos $\left(\mathrm{w}_{\mathrm{v}}\right)$ de um fator num intervalo de unidade decimal. A tabela 2 apresenta o exemplo das ponderações $w_{\mathrm{v}}$ e $w_{\mathrm{F}}$ para o fator 4 sob ponderação sistemática, quando o numero de fatores for igual a 5 . Essa tabela se repete para os demais fatores $(1,2,3$ e 5) quando estes estão sob ponderação sistemática.

Tabela 2. Pesos para cinco fatores, com o fator 4 estando sob ponderação sistemática.

Table 2. Weights for five factors, as the factor 4 is under systematic weighing.

\begin{tabular}{lcccccc}
\hline 5 fatores & \multicolumn{7}{c}{ Peso } \\
\hline Fator 1 fixo & $\mathrm{w}_{\mathrm{F}}=$ & 0,225 & 0,2 & 0,175 & 0,15 & 0,125 \\
Fator 2 fixo & $\mathrm{w}_{\mathrm{F}}=$ & 0,225 & 0,2 & 0,175 & 0,15 & 0,125 \\
Fator 3 fixo & $\mathrm{w}_{\mathrm{F}}=$ & 0,225 & 0,2 & 0,175 & 0,15 & 0,125 \\
Fator 4 sob ponderação & $\mathbf{w}_{\mathbf{v}}=$ & $\mathbf{0 , 1}$ & $\mathbf{0 , 2}$ & $\mathbf{0 , 3}$ & $\mathbf{0 , 4}$ & $\mathbf{0 , 5}$ \\
sistemática & $\mathrm{w}_{\mathrm{F}}=$ & 0,225 & 0,2 & 0,175 & 0,15 & 0,125 \\
Fator 5 fixo & $\Sigma$ & 1 & 1 & 1 & 1 & 1 \\
& & & & &
\end{tabular}

Após os pesos serem atribuídos a todos os fatores, obtém-se um conjunto ponderado de $n$ fatores vezes $p$ pesos. A partir desse conjunto, é feita a análise de sensibilidade, obtida pela correlação entre um cenário homogêneo - em que os pesos dos fatores são iguais - e os demais cenários ponderados sistematicamente.

Uma vez que em uma análise multicritério a soma dos pesos atribuídos deve resultar no valor 1 (um), conforme demonstra a Equação 1, quando se tem apenas dois fatores no conjunto não há sentido na realização da sua análise de sensibilidade de forma individual, pois os pesos serão distribuídos de maneira que se complementem mutuamente. Assim, para os aspectos áreas de mineração (fatores títulos minerários e fragilidade dos cursos d'água) e fragilidade à erosão (fatores solo e declividade), apresentados na tabela 1, não será realizada a análise de sensibilidade de forma individual.

Portanto, para aspecto geologia e geomorfologia, a análise de sensibilidade compreende os fatores água subterrânea (A), geologia (B), geomorfologia (C), aquífero (D), superfície potenciométrica (E) e zona vadosa (F). Para a composição do aspecto estabilidade de encostas, foram definidos os fatores declividade $(\mathrm{G})$, geologia $(\mathrm{H})$ e geomorfologia (I). O aspecto contaminação das águas constitui-se dos 
fatores carga orgânica das populações $(\mathrm{J})$, carga orgânica dos rebanhos $(\mathrm{K})$ e índice agrícola $(\mathrm{L})$. Além da análise de sensibilidade realizada para cada aspecto separadamente, há a necessidade de realizar a análise levando-se em conta todo o conjunto de aspectos, que neste estudo foi denominado como bloco físico (geologialgeomorfologia $(\mathrm{M})$, contaminação das águas $(\mathrm{N})$, estabilidade de encosta $(\mathrm{O})$, áreas de mineração $(\mathrm{P})$ e fragilidade à erosão $(\mathrm{Q}))$.

Correspondência: cenário homogêneo x cenários ponderados

Para a comparação dos cenários construídos através da ponderação, há a premissa estatística da determinação da correspondência entre o cenário testemunha (cenário homogêneo) e os cenários ponderados. Os valores resultantes dessa comparação indicam quais cenários ponderados são mais discriminantes que o cenário testemunha $\mathrm{C}_{\mathrm{H}}$.

Considerando-se que os cenários são imagens digitais, a correspondência diz respeito à medida de associação entre o valor esperado (nas células numéricas da imagem relativa ao $\mathrm{C}_{\mathrm{H}}$ ) e o valor sob teste (nas células equivalentes das imagens ponderadas), da mesma forma encontrada em uma tabela de contingência.

Para efetuar a estatística, utilizou-se o coeficiente de correlação de Pearson disponível na rotina Spatial Analist Tools/Multivariate/Band Collections Statistics, no software ArcGIS 9.3.1, o qual é aplicado às geometrizes numéricas correspondentes aos mapas digitais georreferenciados da área de estudo.

O cenário homogêneo $C_{H}$ caracteriza-se por não priorizar nenhum dos temas de sua composição, sendo por isso a situação menos desejável em uma ponderação multicriterial. Portanto, o método para testar a sensibilidade consiste em comparar as matrizes (rasters) sistematicamente ponderadas, a fim de, diferentemente dos casos estatísticos clássicos, em que se busca a aproximação a uma curva de tendência, encontrar as composições que mais se afastem dessa tendência. Em outras palavras, ao adotar o cenário homogêneo como testemunha (ou "cenário esperado"), os resultados de uma análise de sensibilidade são tão mais convenientes (desejáveis) quanto mais afastados da tendência testemunhal, explicando-se dessa forma a inversão estatística.

\section{Resultados e discussão}

Para todos os aspectos foram atribuídas as ponderações dos cenários $\left(C_{1}\right.$ a $\left.C_{5}\right)$ e posteriormente realizada a análise de correlação com o respectivo cenário homogêneo $\left(\mathrm{C}_{\mathrm{H}}\right)$ do aspecto, resultando nos valores apresentados na tabela 3 .

Os resultados foram convertidos em tabela e gráficos de ponderação $\left(\mathrm{C}_{\mathrm{H} 1}\right.$ a $\left.\mathrm{C}_{\mathrm{H} 5}\right)$ x correlação (0 a 1), para melhor visualização. $O$ resultado da correlação entre o cenário homogêneo $\left(C_{H}\right)$ e o cenário ponderado sob a variação do fator $1\left(\mathrm{C}_{1}\right)$ denomina-se $\mathrm{C}_{\mathrm{H} 1}$, seguindo o raciocínio para os demais cenários ponderados. Os pesos para cada cenário e as correlações do $\mathrm{C}_{\mathrm{H}}$ com cada um dos cenários ponderados dos fatores seguiram conforme os valores apresentados na tabela 3 .

Os resultados de correlação são apresentados em gráficos nos itens a seguir. Nestes gráficos, cada curva representa a distribuição dos valores de correlação à medida que se alteram os pesos atribuídos aos fatores.

A interpretação geral dos gráficos é que as ponderações associadas a altos valores de correlação - que representam maior aproximação com a homogeneidade - não são as mais adequadas. Em oposição, os cenários menos correlacionados com o cenário homogêneo são aqueles em que a ponderação multicriterial apresenta as melhores discriminâncias do ponto de vista do geoprocessamento, configurando-se como as situações mais desejáveis. Essa interpretação é realizada tanto de maneira individual quanto no conjunto dos fatores.

A discussão dos resultados referir-se-á à interpretação dos comportamentos entre as ponderações sistemáticas atribuídas aos fatores em relação aos aspectos e destes em relação ao bloco quando comparadas as suas respectivas ponderações homogêneas, tendo-se como indicador estatístico de sensibilidade o coeficiente de correlação de Pearson.

\section{Geologia e geomorfologia}

Esse aspecto apresentou pouca discriminância entre os fatores, pois todos mantiveram uma correlação considerada alta na amplitude dos pesos atribuídos.

Na figura 3, os fatores aquífero e água subterrânea apresentaram comportamento praticamente similar, podendo posteriormente ser utilizado apenas um desses fatores, ficando ao tomador de decisão a opção entre manter ambos ou escolher o que permanecerá no modelo. Nesse caso em que os fatores 
apresentam comportamentos similares, pode-se levar ao descarte do fator com maior dificuldade na obtenção de dados.

Tabela 3. Pesos atribuídos aos fatores dos cenários e correlação do cenário homogêneo x cenários ponderados.

Table 3. Weights assigned to the factors of the scenarios and correlation of the homogeneous scenario versus weighted scenarios.

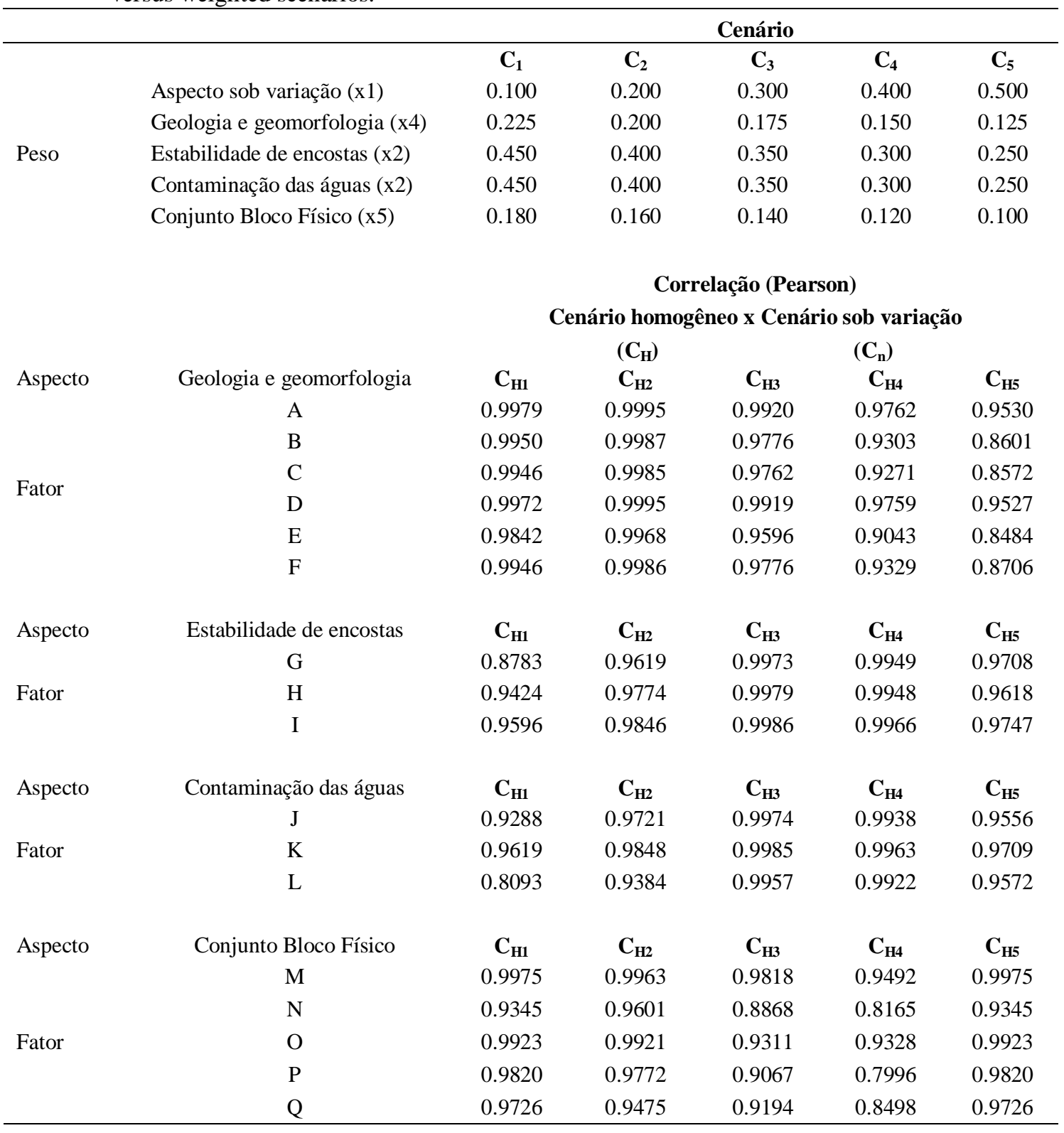

Os fatores geologia, geomorfologia e zona vadosa também apresentaram comportamento bastante similar, aplicando-se o mesmo raciocínio. No entanto, o fator superfície potenciométrica apresentou comportamento único, embora demonstre a mesma curva de tendência das anteriores. Nos pesos 0,3 e 0,4, esse fator apresentou um maior distanciamento em relação aos anteriores, tornando-se distinto dos demais.

Uma sugestão para esse aspecto é que se evite atribuir pesos próximos ou menores do que o valor 0,25 , uma vez que se aproximam do cenário homogêneo. Isso enfatiza a necessidade da redução na 
quantidade de fatores que compõem o aspecto, pois se houver a atribuição individual de pesos acima desse valor, o somatório excede o valor a ser distribuído, conforme a equação 2 .

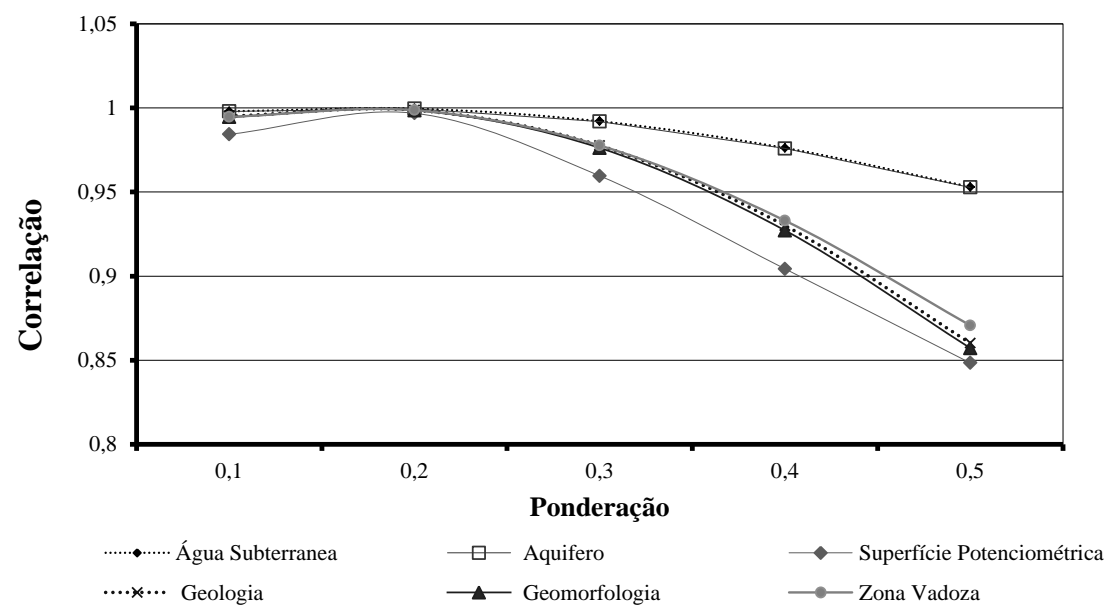

Figura 3. Resultado da análise de sensibilidade para o aspecto geologia e geomorfologia.

Figure 3. Results of the sensitiveness analysis for the geology and geomorphology aspects.

A análise do conjunto dos fatores sugere que o aspecto pode ser construído com três fatores, dadas as similaridades correlacionais entre alguns deles, sendo que variável fator superfície potenciométrica, por apresentar comportamento único, teria a preferência quanto à permanência no modelo.

\section{Estabilidade de encostas}

Ao analisar a figura 4, é possível observar que todas as curvas do gráfico apresentaram a mesma tendência de distribuição, demonstrando o mesmo valor de importância para cada fator à medida que se diminui o valor do peso a ser atribuído. O gráfico demonstra que a atribuição de um peso alto para um determinado fator nem sempre resulta na sua melhor representatividade em relação aos demais.

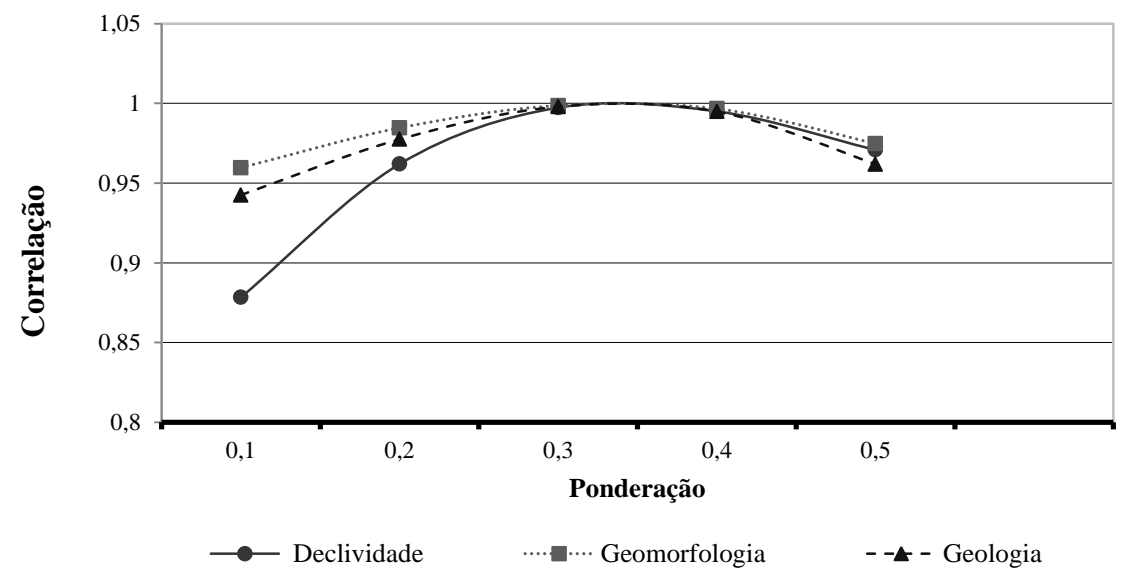

Figura 4. Resultado da análise e de sensibilidade para o aspecto estabilidade de encosta.

Figure 4. Results of the sensitiveness analysis for the slope stability aspect.

Pode-se observar que, apesar de apresentar uma correlação considerada alta para este modelo de análise, a distribuição de pesos que se considera mais discriminante está localizada abaixo do valor de 0,25 , sugerindo a não utilização dos valores entre 0,3 e 0,4 . 
Essa avaliação permite ao(s) decisor(es) discutir se é necessária a utilização dos fatores geologia e geomorfologia, uma vez que os dois apresentaram uma correlação estatisticamente semelhante. Essa interferência do tomador de decisão, segundo Francisco et al. (2008), é importante em casos nos quais haja algumas distorções na análise, utilizando assim a sua experiência como complemento aos resultados obtidos pelas análises. No entanto, Martins (2009) recomenda a busca de um equilíbrio entre a avaliação dos critérios pelos especialistas da área e por não especialistas, para que não haja tendenciosidade nos pesos dos critérios e, consequentemente, uma inconsistência nas análises.

Quanto ao fator declividade, ele apresenta maior discriminância quando lhe é atribuído um peso até no máximo 0,15 .

\section{Contaminação das águas}

Apresentou a mesma tendência dos dados observada no item anterior, ou seja, também apresentaram uma tendência de diminuir a discriminância à medida que o peso atribuído é aumentado.

$\mathrm{Na}$ tabela 2 pode-se observar que a variável fator índice agrícola, apesar de apresentar uma correlação considerada alta, manteve maior discriminância que os demais fatores quando atribuídos pesos abaixo do valor de 0,15 . Em decorrência disso, pode-se sugerir que esse fator receba o menor peso no conjunto de fatores, já que os demais não apresentaram grande amplitude nos resultados à medida que foram alterados os pesos.

\section{Conjunto dos aspectos componentes do bloco físico}

Pela análise das curvas apresentadas pelo gráfico na figura 5, pode-se inferir que o bloco físico apresentou altas correlações na maioria dos casos. O gráfico apresentou uma distribuição variada entre as curvas, sendo que essa distribuição (comportamento) das curvas permite que o tomador de decisão tenha mais liberdade na escolha e determinação de quais aspectos são mais representativos no modelo que se deseja. Com relação a estes, pode-se destacar que, para os aspectos áreas de mineração, contaminação das águas e fragilidade à erosão, o gráfico sugere a utilização de pesos acima de 0,4 , pois é a partir desse valor que esses aspectos apresentam a menor correlação.

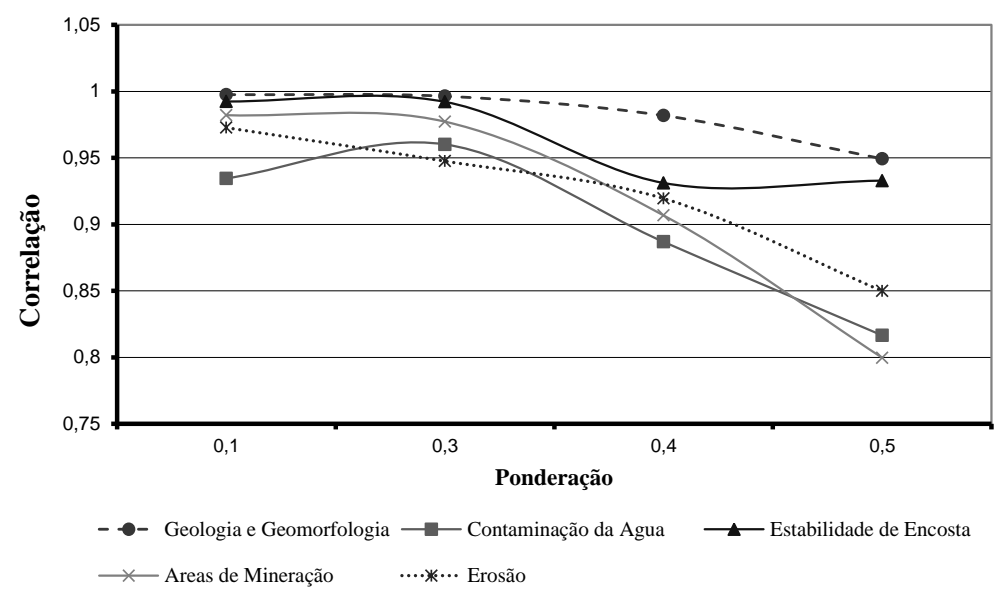

Figura 5. Resultado da análise de sensibilidade para o bloco físico.

Figure 5. Results of the sensitiveness analysis for the physical block.

No entanto, a elevação do peso de um desses aspectos implicará a redução conjunta dos outros quatro, para atender à soma 1 (um). Nesse caso, o julgamento acerca de qual aspecto receberá o peso de maior valor será em função do objetivo do modelo proposto. Nos casos em que a curva tem tendência decrescente para os pesos menores que 0,1 , é possível considerar a atribuição de pesos baixos, sem que essa escolha remeta a uma desvalorização da importância do aspecto, como no caso do aspecto contaminação das águas.

O aspecto geologia e geomorfologia apresentou pouca variação da correlação, de modo que poderia receber qualquer valor de toda a escala dos pesos atribuídos, ficando a critério dos tomadores de 
decisão definir qual a sua importância dentro do modelo. Já estabilidade de encosta apresentou um declínio quando recebeu um peso próximo de 0,4 , voltando a aumentar quando recebe um valor de peso maior. Em uma análise individual, o peso mais indicado estaria entre os valores de 0,35 e 0,45, entretanto, dadas as considerações anteriores, isso nem sempre é possível.

Essa análise conjunta é importante, pois se pode considerar que cada aspecto está interferindo e/ou recebendo interferência do conjunto, fazendo com que o tomador de decisão não se deixe influenciar apenas pela escolha da ponderação, inclusão ou exclusão de uma variável pela sua análise individual, mas entendendo como aquela variável pode interferir no conjunto do modelo.

\section{CONCLUSÕES}

- Nos processos de tomada de decisão em ambiente participativo, o julgamento e a seleção de alternativas ou ações são intrínsecos ao processo. No entanto, os agentes responsáveis pela tomada de decisão, em geral, possuem pontos de vista divergentes e muitas vezes conflitantes, remetendo a discussões quanto ao juízo de valor aplicado para cada uma das alternativas. Em acréscimo, há estudos que refletem a respeito das subjetividades no momento da ponderação. Contudo, apesar do elevado número de pesquisas, ainda se está distante da definição de critérios e ponderações que sejam capazes de representar com alta confiabilidade a relação entre o modelo e a realidade.

- A avaliação multicritério, por ser um processo construtivo, deve ser constantemente revista. Para tanto, a aplicação da sensibilidade da ponderação nessa avaliação, principalmente quando em estudos ambientais, é de fundamental importância, para minimizar os efeitos causados pela adoção de pesos inadequados ou que não representam a verdadeira importância do fator proposto, devendo-se identificar os fatores cujos pesos levaram a uma maior discriminância no conjunto, e que podem, por exemplo, reduzir "emotividades" no momento da definição da importância do peso no contexto da análise.

- Agrupamentos de fatores cujas linhas apresentam a mesma tendência no gráfico (curvatura) indicam semelhança correlacional, levando à possibilidade de uma redução no número dos fatores propostos inicialmente. Nesse caso, faz-se a opção pela permanência do fator que apresenta a maior qualidade e confiabilidade dos dados de origem, ou seja, aquele que apresenta a menor generalização temática. Se isso não for possível, busca-se um índice que correlacione a importância dos fatores entre si, comparando-os em pares, podendo este ser utilizado para a decisão de qual permanecerá no modelo.

- A análise de sensibilidade permitiu o refinamento de uma avaliação multicritério, pelo fato de agregar maior consistência na avaliação de um conjunto de dados em um modelo de análise ambiental. O processo de aplicação dessa análise para o ajuste quali-quantitativo dos fatores se faz importante na medida em que, após o seu processamento, fica mais evidente a identificação da influência de cada fator dentro do conjunto.

- Deve-se observar que a sensibilidade de um fator não necessariamente terá a mesma tendência para regiões diferentes daquela em que foi realizado o estudo, carecendo, portanto, que novas pesquisas sejam realizadas. Assim, os resultados dessa abordagem demonstram a importância da aplicação do estudo de sensibilidade nos modelos de fragilidade ambiental, contribuindo para a compreensão da influência dos fatores no processo decisório, por conferir maior confiabilidade no momento em que apresenta diferentes alternativas de decisão.

- Uma vez que esses modelos têm como objetivo representar cenários alternativos, buscando reduzir a distância comparativa entre eles e a realidade, a análise de sensibilidade foi útil em seu propósito principal, por apresentar alternativas na composição da ponderação constituinte do modelo proposto. Sugere-se aplicar o método descrito neste artigo aos demais aspectos e blocos que compreendem a modelagem ambiental da bacia hidrográfica - os blocos bióticos e antrópico -, sendo então possível compreender todos os parâmetros e variáveis constituintes.

- Considerando a importância de novos paradigmas na elaboração de modelos ambientais, esta pesquisa buscou ampliar o conhecimento e as discussões a respeito da efetiva representatividade da ponderação para convergência entre a modelagem e a realidade, representando para este modelo um importante subsídio à tomada de decisão, ao minimizar as incertezas quanto aos critérios e ponderações integrantes do processo de análise em nível de bacia hidrográfica. 


\section{AGRADECIMENTOS}

Os autores agradecem à equipe do Projeto Fragilidades Ambientais do Rio Uruguai (FRAGRIO) da Universidade Federal de Santa Maria e da Universidade Federal do Pampa, que realizaram a construção das variáveis analisadas neste trabalho, assim como à Coordenação de Aperfeiçoamento de Pessoal de Nível Superior (CAPES), pela bolsa de doutoramento.

\section{REFERÊNCIAS}

BOAS, C. L. V. Análise da aplicação de métodos multicritérios de apoio à decisão (MMAD) na gestão de recursos hídricos. In: SIMPOSIO BRASILEIRO DE RECURSOS HÍDRICOS, 16., 2005, João Pessoa. Anais... João Pessoa: ABRH. 2005. v. 1 CD-ROM.

. Modelo multicritérios de apoio à decisão aplicado ao uso múltiplo de reservatórios: estudo da barragem do ribeirão João Leite. 145 f. Dissertação (Mestrado em Economia) - Universidade de Brasília, Brasília, 2006. Disponível em <http://www.cprm.gov.br/publique/media/vilas_boas.pdf>. Acesso: $02 / 12 / 2013$.

LANG, S.; BLASCHKE, T. Análise de paisagem com SIG. São Paulo. Oficina de Textos. 2009. 424 p.

FERRAZ, S. F. de B.; VETTORAZZI, C. A. Identificação de áreas para recomposição florestal com base em princípios de ecologia de paisagem. Revista Árvore, Viçosa, v. 27, n. 4, p. 575 - 583, 2003.

FERRAZ, S. F. de B.; DE PAULA, F. R.; VETTORAZZI, C. A. Incorporação de indicadores de sustentabilidade na priorização de áreas para restauração florestal na bacia do rio Corumbataí. Revista Árvore, Viçosa, v. 33, n. 5, p. 937 - 947, 2009.

FIDALGO, E. C. C. Critérios para a análise de métodos e indicadores ambientais usados na etapa de diagnóstico de planejamentos ambientais. 276 f. Tese (Doutorado em Engenharia Agrícola) Universidade Estadual de Campinas, Faculdade de Engenharia Agrícola, Campinas, 2003.

FRANCISCO, C. E. S.; COELHO, M. R.; TORRES, R. B.; ADAMI, S. F. Análise multicriterial na seleção de bacia hidrográfica para recuperação ambiental. Revista Ciência Florestal, Santa Maria, v. 18, n. 1, p. 1 - 13, 2008.

MAGANHOTTO, R. F.; SANTOS, L. J. C., OliVEIRA FILHO; P. C. de. Análise da fragilidade ambiental como suporte ao planejamento do ecoturismo em unidades de conservação: estudo de caso Flona de Irati, PR. Revista Floresta, Curitiba v. 41, n. 2, p. 231 - 242, 2011. Disponível em: <http://ojs. c3sl.ufpr.br/ojs2/index.php/floresta/article/view/21871>. Acesso em 09/05/2012.

MARTINS, V. B. Metodologia baseada em sistemas de informações geográficas e análise multicritério para seleção de áreas prioritárias para construção de um repositório para o combustível nuclear usado. $137 \mathrm{f}$. Tese (Doutorado em Engenharia Nuclear) - Universidade Federal do Rio de Janeiro, Rio de Janeiro, 2009.

MINISTÉRIO DO MEIO AMBIENTE (MMA). Caderno da Região Hidrográfica do Uruguai. Brasília: SRH-MMA, 2006. 128 p.

ORGANIZATION FOR ECONOMIC CO-OPERATION AND DEVELOPMENT (OECD). Environmental Indicators: Towards sustainable Development, 2011. 158 p. Disponível em <http://www.oecd.org/site/worldforum /33703867.pdf >. Acesso 02/12/2013.

ROSS, J. L. S. Análise empírica da fragilidade dos ambientes naturais e antropizados. Revista do Departamento de Geografia, São Paulo, n. 8, p. 63 - 73, 1994.

SILVEIRA, G. L.; CRUZ, J. C.; CRUZ, R. C.; DEWES, R.; ARAÚJO, T. S. Concepção Geral. In: SILVEIRA, G. L.; CRUZ, J. C. Seleção ambiental de barragens: análise de favorabilidades ambientais em escala de bacia hidrográfica. Santa Maria: UFSM, 2005. 388 p.

SANTOS, R. F. Planejamento ambiental: teoria e prática. São Paulo. Oficina de Textos. 2004. 184 p.

$\overline{192 \mathrm{p} .}$

Vulnerabilidade ambiental: desastres naturais ou fenômenos induzidos? Brasília: MMA. 2007. 
TREVISAN, M. L. Sensibilidade de fatores para valoração do ambiente com o uso de avaliação multicritério e geoprocessamento digital. 165 f. Tese (Doutorado em Engenharia Agrícola) Universidade Federal de Santa Maria, Santa Maria, 2008.

UNIVERSIDADE FEDERAL DE SANTA MARIA (UFSM.). Inventário de recursos hídricos e de possíveis locais de barramento para fins de irrigação na parte norte do Rio Grande do Sul. Santa Maria UFSM/MAPA, 2002. Relatório Síntese. 249 p.

UNIVERSIDADE FEDERAL DE SANTA MARIA e UNIVERSIDADE DO PAMPA (UFSM/UNIPAMPA). Desenvolvimento metodológico e tecnológico para avaliação ambiental integrada aplicada ao processo de análise da viabilidade de hidrelétricas. FRAG-RIO - Relatório da Etapa 2, 2011 MMA/FATEC/UNIPAMPA/UFSM, Santa Maria. 270 p.

VETTORAZZI, C. A.; VALENTE, R. O. A.; BALLESTER, M. V. R. Forest fire hazard mapping in a GIS environment for a river basin in the State of São Paulo, Brazil. In: INTERNATIONAL CONFERENCE ON GEOSPATIAL INFORMATION IN AGRICULTURE AND FORESTRY, 2., 2000, Lake Buena Vista. Proceedings... Ann Arbor: ERIM International, v. 1, p. 10 - 12, 2000.

ZUFFO, A. C. Seleção e aplicação de métodos multicriteriais ao planejamento ambiental de recursos hídricos. $111 \mathrm{f}$. Tese (Doutorado em Engenharia Civil) - Universidade de São Paulo. São Carlos, 1998. 